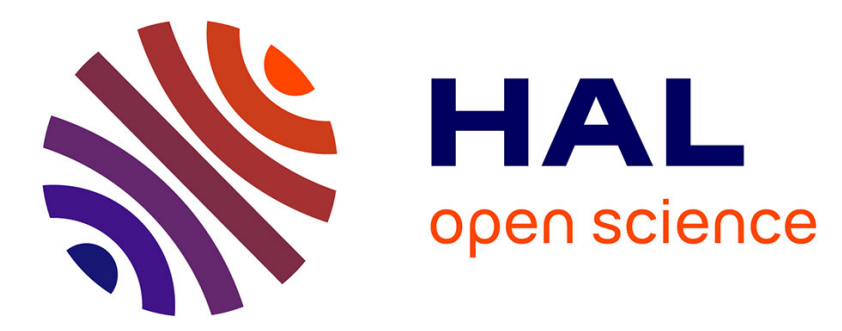

\title{
Spatial correlation in 3D MIMO channels using fourier coefficients of power spectrums
}

Qurrat-Ul-Ain Nadeem, Abla Kammoun, Mérouane Debbah, Mohamed-Slim

Alouini

\section{- To cite this version:}

Qurrat-Ul-Ain Nadeem, Abla Kammoun, Mérouane Debbah, Mohamed-Slim Alouini. Spatial correlation in 3D MIMO channels using fourier coefficients of power spectrums. IEEE wireless communications and networking conference (WCNC), Mar 2015, New Orleans, United States. 10.1109/WCNC.2015.7127463 . hal-01242490

\section{HAL Id: hal-01242490 \\ https://hal.science/hal-01242490}

Submitted on 14 Dec 2015

HAL is a multi-disciplinary open access archive for the deposit and dissemination of scientific research documents, whether they are published or not. The documents may come from teaching and research institutions in France or abroad, or from public or private research centers.
L'archive ouverte pluridisciplinaire HAL, est destinée au dépôt et à la diffusion de documents scientifiques de niveau recherche, publiés ou non, émanant des établissements d'enseignement et de recherche français ou étrangers, des laboratoires publics ou privés. 


\title{
Spatial Correlation in 3D MIMO Channels using Fourier Coefficients of Power Spectrums
}

\author{
Qurrat-Ul-Ain Nadeem*, Abla Kammoun*, Mérouane Debbah ${ }^{\dagger}$, and Mohamed-Slim Alouini* \\ * CEMSE Division, King Abdullah University of Science and Technology (KAUST), Thuwal, Saudi Arabia. \\ Email: \{qurratulain.nadeem, abla.kammoun,slim.alouini\}@kaust.edu.sa \\ $\dagger$ Mathematical and Algorithmic Sciences Lab, Huawei France R\&D, Paris, France. Email: merouane.debbah@ huawei.com
}

\begin{abstract}
In this paper, an exact closed-form expression for the Spatial Correlation Function (SCF) is derived for the standardized three-dimensional (3D) multiple-input multiple-output (MIMO) channel. This novel SCF is developed for a uniform linear array of antennas with non-isotropic antenna patterns. The proposed method resorts to the spherical harmonic expansion (SHE) of plane waves and the trigonometric expansion of Legendre and associated Legendre polynomials to obtain a closed-form expression for the SCF for arbitrary angular distributions and antenna patterns. The resulting expression depends on the underlying angular distributions and antenna patterns through the Fourier Series (FS) coefficients of power azimuth and elevation spectrums. The novelty of the proposed method lies in the SCF being valid for any 3D propagation environment. Numerical results validate the proposed analytical expression and study the impact of angular spreads on the correlation. The derived SCF will help evaluate the performance of correlated 3D MIMO channels in the future.
\end{abstract}

\section{INTRODUCTION}

Multiple-input multiple-output (MIMO) systems have remained a subject of interest in wireless communications over the past decade due to the significant gains they offer in terms of capacity by exploiting the multipath richness of the channel. Pioneer work in this area by Telatar [1] and Foschini [2] realized that capacity can potentially scale linearly with the number of antennas. These MIMO systems were designed to support antenna configurations capable of adaptation in azimuth only. To further enhance the system performance, researchers and industrials are now exploiting the channel's degrees of freedom in the elevation as well. Encouraged by the initial implementations of this technology [3], the 3GPP has been working on defining future mobile communication standards that allow for elevation beamforming [4]. However in realistic propagation environments, the promised gains are not realized due to the spatial correlation present in the MIMO channel. Deriving and simulating this correlation is therefore essential to predict the performance of MIMO systems.

The need to investigate the impact of spatial correlation on the performance of MIMO systems is acknowledged and wellknown among researchers. However, the spatial correlation

The work of Q.-U.-A. Nadeem and A. Kammoun was supported by KAUST. The work of Mérouane Debbah was supported by French pôle de compétitivité SYSTEM@TIC within the project 4G in Vitro and the work of M. -S. Alouini was supported by the Qatar National Research Fund (a member of Qatar Foundation) under NPRP Grant NPRP 4-353-2-130. The statements made herein are solely the responsibility of the authors. models proposed in literature occasionally ignore the elevation dimension and antenna patterns which can cause the simulated results to be misleading. Most of these models are derived for a particular distribution of the Angle of Departure (AoD) and Angle of Arrival (AoA) such as uniform, Gaussian, Von Mises or Laplacian. In [5], approximate closed-form expressions for spatial correlation coefficients were derived for clustered MIMO channel assuming Laplacian azimuth AoA distribution. The proposed method makes small angle spread approximation and offers significant gains in terms of computational cost. In [6], the authors derived exact closed-form expressions for the spatial correlation between receive antenna elements for cosine, Gaussian and Von Mises azimuth AoA distributions. The use of Von Mises was shown to simplify the expressions and the impact of mutual coupling on correlation was studied.

The notion of spatial correlation in three-dimensional (3D) environments has been addressed in some works. The authors in [7] showed that elevation plays a crucial role in determining the Spatial Correlation Function (SCF). The derivation is based on the spherical harmonic expansion (SHE) of plane waves and assumes the distribution of AoA to be 3D Von Mises-Fisher. The effect of non-isotropic antenna patterns was studied using numerical techniques. The analysis in [8] uses SHE to compute generalized expressions for the correlation. Although the tools presented are handy, the proposed method requires certain assumptions to be made on the propagation environment. Even a simple assumption of angles being uniformly distributed resulted in integrals involving Legendre polynomials that could not be expressed in a closed-form. Such assumptions neither aptly represent realistic propagation environments nor make the proposed method truly generic in nature. To the best of authors' knowledge, a SCF that works for the 3D channel without making assumptions on the underlying angular distributions and antenna patterns has not been developed before.

In this paper, we propose a novel method for obtaining a closed-form expression for the SCF for 3D MIMO channels that can be used for any arbitrary choice of antenna patterns and distribution of azimuth and elevation AoD and AoA. The SCF is derived for channel models proposed in standards like 3GPP SCM [9], WINNER+ [10] and ITU [11]. To get an analytically tractable closed-form solution, the SHE of plane waves and properties of Legendre and associated Legendre polynomials are exploited. A uniform linear array of antennas is considered. Power azimuth spectrum (PAS) and power 


$$
[\mathbf{H}]_{s u}=\sum_{n=1}^{N} \alpha_{n} \sqrt{g_{t}\left(\phi_{n}, \theta_{n}, \theta_{t i l t}\right)} \exp \left(i k(s-1) d_{t} \sin \phi_{n} \sin \theta_{n}\right) \sqrt{g_{r}\left(\varphi_{n}, \vartheta_{n}\right)} \exp \left(i k(u-1) d_{r} \sin \varphi_{n} \sin \vartheta_{n}\right)
$$

elevation spectrum (PES) are explicitly defined and their relationships with the respective angular distributions highlighted. The final expression of SCF depends on the channel and antenna parameters through the Fourier Series (FS) coefficients of PAS and PES. The researchers and industrials interested in using our method to obtain the correlation coefficients need to provide only the FS coefficients of the PAS and PES they are using for the evaluation of their work, which can generally be obtained easily for popular angular distributions. The proposed SCF is validated via simulations using angular distributions and antenna patterns specified in the standards and is seen to coincide with the Monte-Carlo simulated results.

The rest of the paper is organized as follows. The 3D standardized channel model, PAS and PES are discussed in Section II. The proposed SCF is derived in section III. Section IV provides simulation results that validate the proposed function and section $\mathrm{V}$ concludes the paper.

\section{Channel Model and Power Spectrums}

Encouraged by the potential of elevation beamforming to enhance system performance, many standardized channel models have emerged to define the next generation 3D channels. We base the evaluation of our work on these models while making some realistic assumptions on the channel parameters.

\section{A. Standardized 3D Channel Model}

The mobile communication standards like SCM [9], ITU [11], WINNER [10] follow a system level, stochastic channel modeling approach wherein, the propagation paths are described through statistical parameters, like delay, amplitude, AoA and AoD. The extensions of these 2D models to the 3D case have started to emerge recently [10], [4].

The antenna configuration we consider is shown in Fig. 1. There are $\mathrm{N}_{B S}$ and $\mathrm{N}_{M S}$ antenna ports at the base station (BS) and mobile station (MS) respectively. Each antenna port comprises of $M$ vertically stacked antenna elements that determine the effective antenna port pattern. The antenna ports are placed at fixed positions along $\hat{\mathbf{e}}_{y}$, with the elements in each port aligned along $\hat{\mathbf{e}}_{z}$. The transmit (Tx) signal is fed to all elements with corresponding weights $\mathrm{w}_{m}\left(\theta_{\text {tilt }}\right), m=1, \ldots, M$, in order to achieve the desired directivity. The MS sees each antenna port as a single antenna because all the elements carry the same signal. For this antenna configuration, the channel coefficient between BS antenna port $s$ and MS antenna port $u$ is given by (1) [10], [12], where $\phi_{n}$ and $\theta_{n}$ are the azimuth and elevation AoD of the $n^{\text {th }}$ path respectively, $\varphi_{n}$ and $\vartheta_{n}$ are the azimuth and elevation AoA of the $n^{t h}$ path respectively, $\theta_{t i l t}$ is the tilt angle, which plays a crucial role in elevation beamforming strategies and $\alpha_{n}$ is the complex amplitude of the $n^{t h}$ path. The complex amplitudes are assumed to be i.i.d zero

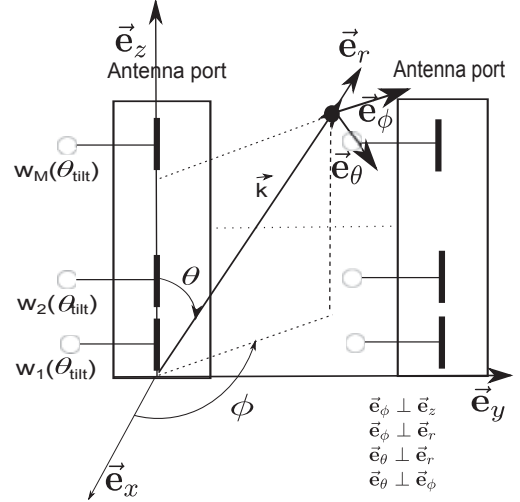

Fig. 1. Antenna configuration.

mean, $\frac{1}{N}$ variance Normal RVs. Also $\sqrt{\mathrm{g}_{t}\left(\phi_{n}, \theta_{n}, \theta_{t i l t}\right)}$ and $\sqrt{\mathrm{g}_{r}\left(\varphi_{n}, \vartheta_{n}\right)}$ are the global patterns of Tx and Rx antennas respectively where $g_{t}\left(\phi_{n}, \theta_{n}, \theta_{t i l t}\right) \approx g_{t, H}\left(\phi_{n}\right) g_{t, V}\left(\theta_{n}, \theta_{t i l t}\right)$ and $g_{r}\left(\varphi_{n}, \vartheta_{n}\right) \approx g_{r, H}\left(\varphi_{n}\right) g_{r, V}\left(\vartheta_{n}\right) \cdot g_{t, H}(\phi), g_{r, H}(\varphi)$ are the horizontal antenna patterns and $g_{t, V}\left(\theta, \theta_{t i l t}\right), g_{r, V}(\vartheta)$ are the vertical antenna patterns. $d_{t}$ and $d_{r}$ are the separations between Tx antenna ports and $\mathrm{Rx}$ antenna ports respectively and $k$ is the wave number that equals $\frac{2 \pi}{\lambda}$, where $\lambda$ is the wavelength of the carrier frequency. Also the entries [12],

$$
\begin{aligned}
{\left[\mathbf{a}_{t}(\phi, \theta)\right]_{s} } & =\exp \left(i k(s-1) d_{t} \sin \phi \sin \theta\right) \text { and } \\
{\left[\mathbf{a}_{r}(\varphi, \vartheta)\right]_{u} } & =\exp \left(i k(u-1) d_{r} \sin \varphi \sin \vartheta\right)
\end{aligned}
$$

are the array responses of $s^{t h} \mathrm{Tx}$ and $u^{\text {th }} \mathrm{Rx}$ antennas respectively. Fig. 2 shows the 3D channel model being considered.

\section{B. Power Azimuth and Elevation Spectrums}

PAS and PES are important statistical properties of wireless channels and will play an important role in deriving the SCF. They provide a measure of the power distribution upon the azimuth AoD and AoA and elevation AoD and AoA respectively. Observing that the integral of the product of

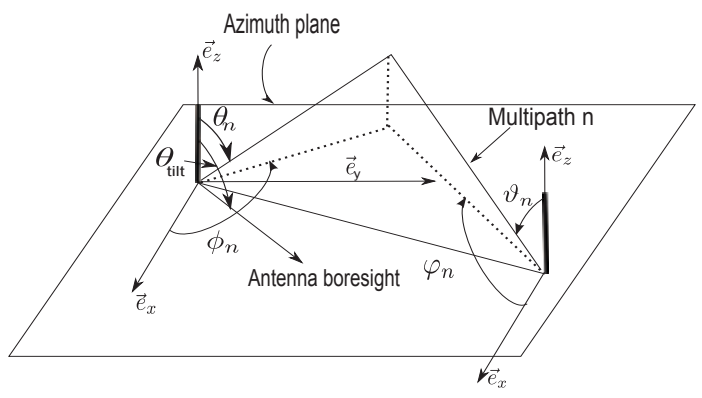

Fig. 2. 3D channel model. 


$$
\begin{gathered}
P_{n}(\cos \gamma)=P_{n}\left(\cos \theta_{1}\right) P_{n}\left(\cos \theta_{2}\right)+2 \sum_{m=1}^{n} \frac{(n-m) !}{(n+m) !} P_{n}^{m}\left(\cos \theta_{1}\right) P_{n}^{m}\left(\cos \theta_{2}\right) \cos \left[m\left(\phi_{1}-\phi_{2}\right)\right] \\
\exp \left(i \frac{2 \pi}{\lambda} d_{t}\left(s-s^{\prime}\right) \sin \phi \sin \theta\right)=\sum_{n=0}^{\infty} i^{n}(2 n+1) j_{n}\left(\frac{2 \pi}{\lambda} d_{t}\left|s-s^{\prime}\right|\right) P_{n}(\sin \phi \sin \theta)
\end{gathered}
$$

azimuth angular power density function and the horizontal antenna pattern yields the expected power in the azimuth plane, we define PAS at the transmitter as follows,

$$
\operatorname{PAS}_{t}(\phi)=g_{t, H}(\phi) p_{\phi}(\phi),
$$

where the angular power density function $p_{\phi}(\phi)=f_{\phi}(\phi)$, the probability density function of azimuth angle. Therefore,

$$
\int_{-\pi}^{\pi} p_{\phi}(\phi) d \phi=1
$$

Similarly PES at the Tx side is defined as,

$$
\operatorname{PES}_{t}\left(\theta, \theta_{t i l t}\right)=g_{t, V}\left(\theta, \theta_{t i l t}\right) p_{\theta}(\theta) .
$$

The elevation angular power density function, $p_{\theta}(\theta)=\frac{f_{\theta}(\theta)}{\sin (\theta)}$, which implies [13],

$$
\int_{0}^{2 \pi} p_{\theta}(\theta) \sin (\theta) d \theta=1 .
$$

The same definitions and conditions can be extended to $\mathrm{PAS}_{r}$ and $\mathrm{PES}_{r}$. Note that the limits taken in $(7)$ are $(0,2 \pi)$ instead of $(0, \pi)$, which is the range over which $\theta$ is defined. This extension in limits, which would later assist in expressing SCF in terms of the FS coefficients of PES, entails that we define $f_{\theta}(\theta)$ to be approximately 0 from $\pi$ to $2 \pi$. This is generally true because the elevation angular density spectrum used in standards decays exponentially with $\theta$.

\section{Proposed Spatial Correlation Function And RELATIONSHIP WITH FS COEFFICIENTS OF PAS AND PES}

In this section, we make use of the SHE of plane waves and properties of Legendre and associated Legendre polynomials to derive a generalized analytical expression for the SCF. Before delving into the derivation, we note that in a 3D propagation environment, the array responses of $\mathrm{Tx}$ and $\mathrm{Rx}$ antennas can be expanded using spherical decomposition of plane waves. Using the Jacobi-Anger expansion, a plane electromagnetic wave can be represented by a superposition of spherical waves as [14],

$$
e^{i k \mathbf{x} \cdot \hat{\mathbf{v}}}=\sum_{n=0}^{\infty} i^{n}(2 n+1) j_{n}(k\|\mathbf{x}\|) P_{n}(\hat{\mathbf{x}} \cdot \hat{\mathbf{v}}), \mathbf{x} \in \mathbb{R}^{3},
$$

where $\hat{\mathbf{v}}$ is a unit vector in the direction of wave propagation, $\mathbf{x}$ is a vector in $\mathbb{R}^{3}, j_{n}$ is the spherical Bessel function of order $n$ and $P_{n}$ is the Legendre polynomial function of order $n$.

We also state here the Legendre addition theorem for spherical harmonics [14], [15] which will be employed later in the derivation of the SCF. If $\gamma$ is defined such that,

$$
\cos \gamma=\cos \theta_{1} \cos \theta_{2}+\sin \theta_{1} \sin \theta_{2} \cos \left(\phi_{1}-\phi_{2}\right),
$$

where $\left(\theta_{1}, \phi_{1}\right)$ and $\left(\theta_{2}, \phi_{2}\right)$ are the spherical coordinates of the vectors $\hat{\mathbf{v}}$ and $\mathbf{x}$ respectively in (8), then the Legendre polynomial of argument $\cos (\gamma)$ is given by (10), where $P_{n}^{m}$ are the associated Legendre polynomials.

\section{A. Expansion of SCF}

These results will now be employed to derive a closed-form expression for the SCF. To this end, we observe from (1) that for i.i.d zero mean, $\frac{1}{N}$ variance, $\alpha$ 's, the spatial correlation between the channels constituted by any pair of Tx and Rx antenna ports can be expressed as a product of the correlation between $\mathrm{Tx}$ antenna ports and the correlation between $\mathrm{Rx}$ antenna ports as,

$$
\mathrm{SCF}=\mathbb{E}\left[\mathbf{H}_{s u} \mathbf{H}_{s^{\prime} u^{\prime}}^{H}\right]=\rho_{t}\left(s-s^{\prime}\right) \rho_{r}\left(u-u^{\prime}\right),
$$

where,

$$
\begin{aligned}
& \rho_{t}\left(s-s^{\prime}\right)=\mathbb{E}\left[g_{t}\left(\phi, \theta, \theta_{t i l t}\right) \exp \left(i \frac{2 \pi}{\lambda} d_{t}\left(s-s^{\prime}\right) \sin \phi \sin \theta\right)\right], \\
& \rho_{r}\left(u-u^{\prime}\right)=\mathbb{E}\left[g_{r}(\varphi, \vartheta) \exp \left(i \frac{2 \pi}{\lambda} d_{r}\left(u-u^{\prime}\right) \sin \varphi \sin \vartheta\right)\right]
\end{aligned}
$$

We derive the closed-form expression for the correlation between Tx antenna ports given in (12). The same approach can be extended to the correlation between Rx antenna ports and the product of the two would yield the closed-form expression for the SCF for the standardized 3D MIMO channel.

The SHE result for plane waves in (8) yields alternate expressions for the array responses of $\mathrm{Tx}$ and $\mathrm{Rx}$ antennas. The Tx array response in (2) can be expanded as a sum of spherical waves as shown in (14). From Fig. 1, the spherical coordinates $\left(\theta_{1}, \phi_{1}\right)$ of the wave vector are $(\theta, \phi)$, and the spherical coordinates $\left(\theta_{2}, \phi_{2}\right)$ of the vector along $d_{t}\left(s-s^{\prime}\right)$ are $\left(\frac{\pi}{2}, \frac{\pi}{2}\right)$. Combining the addition theorem in (10) with (14), such that $\cos \gamma=\sin \phi \sin \theta$ and using this expansion of the array response in the expression of correlation between $\mathrm{Tx}$ antenna ports given in (12) leads to (15). We now use some properties of Legendre and associated Legendre polynomials to expand (15) in a systematic way that would later help us in expressing it in terms of FS coefficients of PAS and PES. A sketch of the proof is provided here for reader's convenience. There are several interesting properties of Legendre polynomials, some of which stated below will be exploited now to 


$$
\begin{gathered}
\rho_{t}\left(s-s^{\prime}\right)=\mathbb{E}\left[g _ { t } ( \phi , \theta , \theta _ { t i l t } ) \sum _ { n = 0 } ^ { \infty } i ^ { n } ( 2 n + 1 ) j _ { n } ( \frac { 2 \pi } { \lambda } d _ { t } | s - s ^ { \prime } | ) \left(P_{n}(\cos \theta) P_{n}(0)\right.\right. \\
\left.\left.+2 \sum_{m=1}^{n} \frac{(n-m) !}{(n+m) !} P_{n}^{m}(\cos \theta) P_{n}^{m}(0) \cos \left(m\left(\phi-\frac{\pi}{2}\right)\right)\right)\right] \\
\rho_{t}\left(s-s^{\prime}\right)=\mathbb{E}\left[g_{t}\left(\phi, \theta, \theta_{t i l t}\right)\right] j_{0}\left(\beta_{t}\left|s-s^{\prime}\right|\right)+\sum_{n=1}^{\infty}(-1)^{n}(4 n+1) j_{2 n}\left(\beta_{t}\left|s-s^{\prime}\right|\right) P_{2 n}(0) \mathbb{E}\left[P_{2 n}(\cos \theta) g_{t, V}\left(\theta, \theta_{t i l t}\right)\right] \mathbb{E}\left[g_{t, H}(\phi)\right] \\
+\sum_{n=1}^{\infty} 4(-1)^{n} j_{2 n}\left(\beta_{t}\left|s-s^{\prime}\right|\right)\left(\sum_{m=1}^{n}(-1)^{m} \bar{P}_{2 n}^{2 m}(0) \mathbb{E}\left[\bar{P}_{2 n}^{2 m}(\cos \theta) g_{t, V}\left(\theta, \theta_{t i l t}\right)\right] \mathbb{E}\left[\cos (2 m \phi) g_{t, H}(\phi)\right]\right) \\
+\sum_{n=1}^{\infty} 4 i(-1)^{n} j_{2 n-1}\left(\beta_{t}\left|s-s^{\prime}\right|\right)\left(\sum_{m=1}^{n}(-1)^{m} \bar{P}_{2 n-1}^{2 m-1}(0) \mathbb{E}\left[\bar{P}_{2 n-1}^{2 m-1}(\cos \theta) g_{t, V}\left(\theta, \theta_{t i l t}\right)\right] \mathbb{E}\left[\sin ((2 m-1) \phi) g_{t, H}(\phi)\right]\right)
\end{gathered}
$$

get a closed-form expression for the SCF.

$$
\begin{aligned}
P_{n}(0) & =0, \quad n=1,3,5, \ldots \\
P_{n}^{m}(0) & =0, \quad n+m=1,3,5, \ldots \\
P_{0}(x) & =1 .
\end{aligned}
$$

Before further analysis of the SCF, we define $\bar{P}_{n}^{m}(\mathrm{x})=\sqrt{\left(n+\frac{1}{2}\right) \frac{(n-m) !}{(n+m) !}} P_{n}^{m}(\mathrm{x})$ and denote $\frac{2 \pi}{\lambda} d_{t}$ as $\beta_{t}$. Now using the decomposition of antenna patterns in azimuth and elevation i.e. $g_{t}\left(\phi, \theta, \theta_{t i l t}\right) \approx g_{t, H}(\phi) g_{t, V}\left(\theta, \theta_{t i l t}\right)$, and the properties just described, we expand (15) and take the deterministic terms out of the expectation operator. After some trigonometric manipulations, we get (19). The same approach would yield a similar expression for $\rho_{r}\left(u-u^{\prime}\right)$ with $g_{t}\left(\phi, \theta, \theta_{\text {tilt }}\right)$ replaced by $g_{r}(\varphi, \vartheta)$ and the AoDs replaced by AoAs. The expansion looks alarming at first sight but it will now be shown to yield an interesting closed-form expression that works for any arbitrary choice of antenna patterns and angular distributions.

\section{B. Closed-form Expression of SCF using FS Coefficients of $P A S$ and PES}

The expansion in (19) exhibits several difficulties in deriving a closed-form expression for the SCF. The random variables, AoD and AoA, with respect to which the expectations need to be computed appear as the arguments of Legendre and associated Legendre polynomials. We need a general representation of these polynomials in terms of their arguments that can be used to facilitate the development of the expectation terms. For this purpose, we use the trigonometric expansions of these polynomials that were presented in [16]. They express Legendre and associated Legendre polynomials with even and odd orders as a linear combination of cosines and sines as,

$$
\begin{aligned}
P_{2 n}(\cos x) & =p_{n}^{2}+2 \sum_{k=1}^{n} p_{n-k} p_{n+k} \cos (2 k x),(20) \\
\bar{P}_{2 n}^{2 m}(\cos x) & =\sum_{k=0}^{n} c_{2 n, 2 k}^{2 m} \cos (2 k x)
\end{aligned}
$$

$$
\bar{P}_{2 n-1}^{2 m-1}(\cos x)=\sum_{k=1}^{n} d_{2 n-1,2 k-1}^{2 m-1} \sin ((2 k-1) x) .
$$

The coefficients $p_{n}, c_{2 n, 2 k}^{2 m}$ and $d_{2 n-1,2 k-1}^{2 m-1}$ are generated using the recursion relations provided in [16] (equations 2.8, 3.13.5). Using the relations given in (20)-(22), the expectations in (19) can be expressed analytically as a linear combination of the FS coefficients of PAS and PES. This is made more explicit by defining the FS coefficients of PAS and PES given in (4) and (6) respectively as,

$$
\begin{gathered}
a_{\phi}(m)=\frac{1}{\pi} \int_{-\pi}^{\pi} \operatorname{PAS}_{t}(\phi) \cos (m \phi) d \phi, \\
b_{\phi}(m)=\frac{1}{\pi} \int_{-\pi}^{\pi} \operatorname{PAS}_{t}(\phi) \sin (m \phi) d \phi, \\
a_{\theta}(k)=\frac{1}{\pi} \int_{0}^{2 \pi} \operatorname{PES}_{t}\left(\theta, \theta_{t i l t}\right) \cos (k \theta) d \theta, \\
b_{\theta}(k)=\frac{1}{\pi} \int_{0}^{2 \pi} \operatorname{PES}_{t}\left(\theta, \theta_{t i l t}\right) \sin (k \theta) d \theta .
\end{gathered}
$$

With all these tools in hand, we now present in (27) and (28) the analytical expressions for the correlation between Tx antenna ports and Rx antenna ports respectively in terms of the FS coefficients of PAS and PES. The product of the two yields the SCF as given in (11). The infinite summation over $n$ can be truncated to a small finite number, $N_{0}$, of terms such that the truncation error has a bound that decreases exponentially as shown in [17]. As few as 15 terms are needed to calculate the correlation with significant accuracy. The expressions show how this unprecedented function requires only the FS coefficients of PAS and PES as per the propagation environment and antenna configuration to return the correlation coefficients.

\section{Numerical Results}

In this section, we provide simulation results to verify our proposed SCF for different angular distributions. Since this paper largely focuses on the guidelines provided in the mobile communication standards used globally, so we validate our 


$$
\begin{aligned}
& \rho_{t}\left(s-s^{\prime}\right)=\pi^{2} a_{\phi}(0) b_{\theta}(1) j_{0}\left(\beta_{t}\left|s-s^{\prime}\right|\right)+\sum_{n=1}^{\infty}(-1)^{n}(4 n+1) j_{2 n}\left(\beta_{t}\left|s-s^{\prime}\right|\right) P_{2 n}(0) \pi^{2} \sum_{k=-n}^{n} p_{n-k} p_{n+k} \frac{1}{2}\left[b_{\theta}(2 k+1)\right. \\
& \left.-b_{\theta}(2 k-1)\right] a_{\phi}(0)+\sum_{n=1}^{\infty} 4(-1)^{n} j_{2 n}\left(\beta_{t}\left|s-s^{\prime}\right|\right)\left(\sum_{m=1}^{n}(-1)^{m} \bar{P}_{2 n}^{2 m}(0) \pi^{2} \sum_{k=0}^{n} c_{2 n, 2 k}^{2 m} \frac{1}{2}\left[b_{\theta}(2 k+1)-b_{\theta}(2 k-1)\right] a_{\phi}(2 m)\right) \\
& +\sum_{n=1}^{\infty} 4 i(-1)^{n} j_{2 n-1}\left(\beta_{t}\left|s-s^{\prime}\right|\right)\left(\sum_{m=1}^{n}(-1)^{m} \bar{P}_{2 n-1}^{2 m-1}(0) \pi^{2} \sum_{k=1}^{n} d_{2 n-1,2 k-1}^{2 m-1} \frac{1}{2}\left[a_{\theta}(2 k-2)-a_{\theta}(2 k)\right] b_{\phi}(2 m-1)\right) \\
& \rho_{r}\left(u-u^{\prime}\right)=\pi^{2} a_{\varphi}(0) b_{\vartheta}(1) j_{0}\left(\beta_{r}\left|u-u^{\prime}\right|\right)+\sum_{n=1}^{\infty}(-1)^{n}(4 n+1) j_{2 n}\left(\beta_{r}\left|u-u^{\prime}\right|\right) P_{2 n}(0) \pi^{2} \sum_{k=-n}^{n} p_{n-k} p_{n+k} \frac{1}{2}\left[b_{\vartheta}(2 k+1)\right. \\
& \left.-b_{\vartheta}(2 k-1)\right] a_{\varphi}(0)+\sum_{n=1}^{\infty} 4(-1)^{n} j_{2 n}\left(\beta_{r}\left|u-u^{\prime}\right|\right)\left(\sum_{m=1}^{n}(-1)^{m} \bar{P}_{2 n}^{2 m}(0) \pi^{2} \sum_{k=0}^{n} c_{2 n, 2 k}^{2 m} \frac{1}{2}\left[b_{\vartheta}(2 k+1)-b_{\vartheta}(2 k-1)\right] a_{\varphi}(2 m)\right) \\
& +\sum_{n=1}^{\infty} 4 i(-1)^{n} j_{2 n-1}\left(\beta_{r}\left|u-u^{\prime}\right|\right)\left(\sum_{m=1}^{n}(-1)^{m} \bar{P}_{2 n-1}^{2 m-1}(0) \pi^{2} \sum_{k=1}^{n} d_{2 n-1,2 k-1}^{2 m-1} \frac{1}{2}\left[a_{\vartheta}(2 k-2)-a_{\vartheta}(2 k)\right] b_{\varphi}(2 m-1)\right)
\end{aligned}
$$

model using the angular distributions and antenna patterns specified in the standards. In the standards, elevation AoD and AoA are drawn from Laplacian density spectrum with parameters, mean $\theta_{0}$ and spread $\sigma$ [10], [18]. Also azimuth AoD and AOA are drawn from the Wrapped Gaussian (WG) density spectrum [11], [10], that can be approximated by Von Mises (VM) distribution [19], [6] as follows,

$$
\mathrm{WG}\left(\mu, \sigma^{2}\right)=\mathrm{VM}(\mu, \kappa), \sigma^{2}=2\left[\log I_{0}(\kappa)-\log I_{1}(\kappa)\right]
$$

where $I_{n}(\mathrm{x})$ is the modified Bessel function of order $n, \mu$ is the mean AoD/AoA and $\frac{1}{\kappa}$ is a measure of dispersion in the azimuth. This equivalence is illustrated in Fig. 3 for different choices of $\sigma$ and $\kappa$. To enable an abstraction of the role played by antenna elements, ITU approximates the combined pattern $g_{t}\left(\phi, \theta, \theta_{\text {tilt }}\right)$ of each port by a narrow beam as [11],

$$
-\min \left\{-\left(g_{t, H}(\phi)+g_{t, V}\left(\theta, \theta_{t i l t}\right)\right), 20\right\} \mathrm{dB},
$$

where $g_{t, H}(\phi)$ and $g_{t, V}\left(\theta, \theta_{t i l t}\right)$ can be approximated as,

$$
g_{t, H}(\phi)=-12\left(\frac{\phi}{\phi_{3 d B}}\right)^{2} \mathrm{~dB}
$$

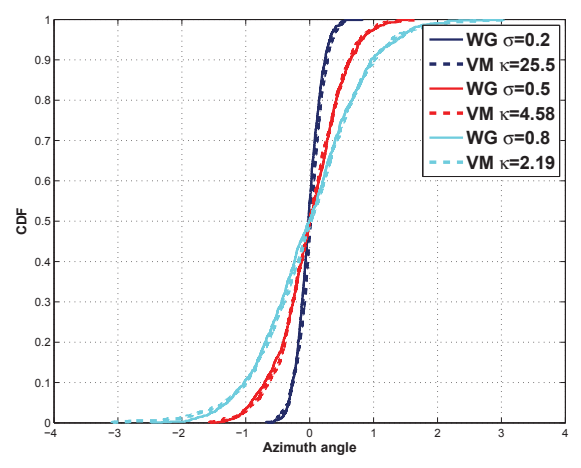

Fig. 3. Equivalence of the Wrapped Gaussian and Von Mises distributions.

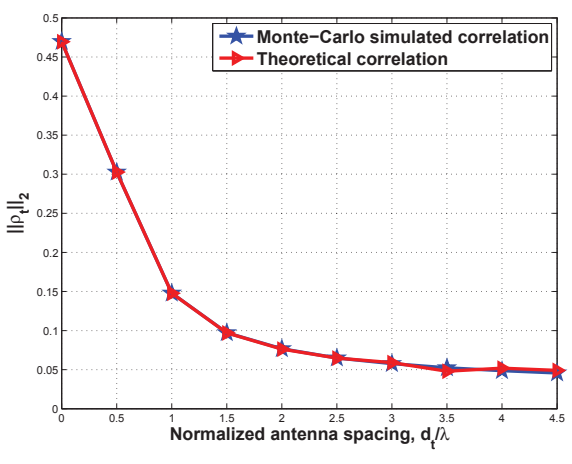

Fig. 4. Correlation between Tx antenna ports using Laplacian elevation and VM azimuth angular distributions and antenna patterns from standards.

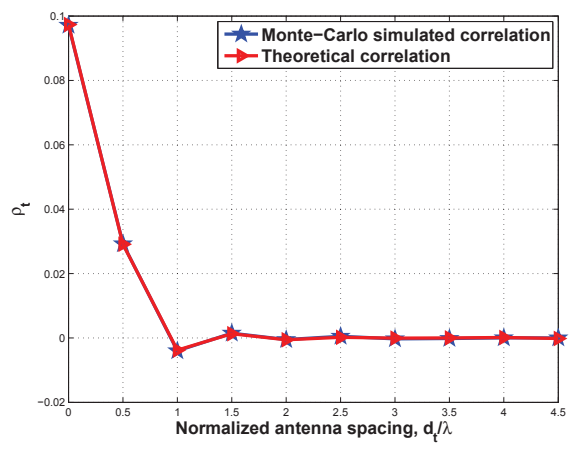

Fig. 5. Correlation between Tx antenna ports using uniform azimuth angular distribution and antenna patterns from standards.

$$
g_{t, V}\left(\theta, \theta_{t i l t}\right)=-12\left(\frac{\theta-\theta_{t i l t}}{\theta_{3 d B}}\right)^{2} \mathrm{~dB}
$$

We set $N_{0}=15, \theta_{\text {tilt }}=95^{\circ}, \theta_{3 d B}=15^{\circ}, \phi_{3 d B}=70^{\circ}$, $\sigma_{s}=7^{\circ}, \theta_{0}=90^{\circ}, \kappa_{s}=5$ and $\mu=\frac{2 \pi}{3}$. A thousand independent Monte-Carlo realizations of the channel are generated to compute (12) for comparison with the theoretical result. The antennas are assumed to be omnidirectional in azimuth to 


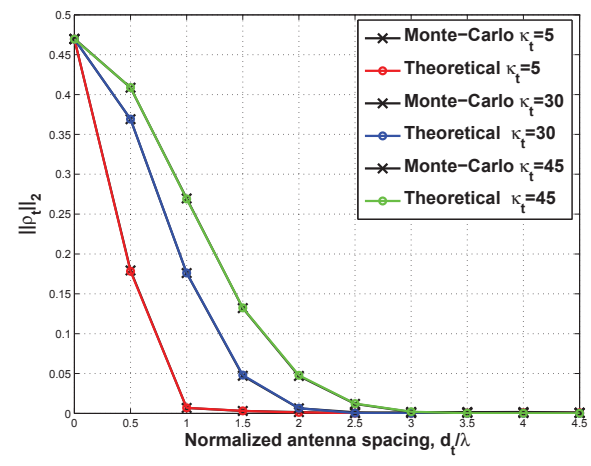

Fig. 6. Effect of the azimuth angular spread on the correlation.

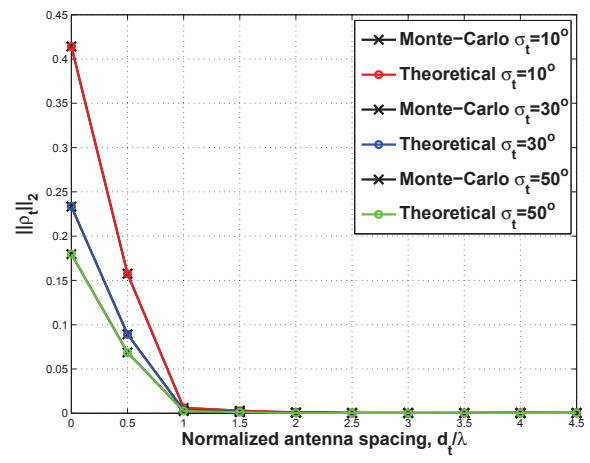

Fig. 7. Effect of the elevation angular spread on the correlation.

simplify the computation of FS coefficients of PAS for VM density. The vertical antenna pattern in (32) is considered. The result is shown in Fig. 4. As expected, the correlation decreases as the distance between antenna ports increases and the theoretical result from (27) coincides with the Monte-Carlo simulated correlation. The results are further validated using uniform azimuth angular distribution and the horizontal pattern in (31). Fig. 5 illustrates the excellent agreement between our derived and Monte-Carlo simulated result and establishes the credibility of the proposed function. Fig. 6 investigates the effect of azimuth angular spread on the correlation between Tx antenna ports using angular distributions from the standards. A decrease in the parameter $\kappa$ for VM distribution corresponds to an increase in the azimuth angular spread that leads to lower values of correlation. An increase in elevation angular spread $\sigma$ also results in lower values of correlation as illustrated in Fig. 7. In fact, small local angular spreads can cause antennas to become highly correlated in the azimuth and elevation.

\section{CONClusion}

In this paper, we characterized the spatial correlation present in a 3D MIMO channel. The conventional SCFs do not take into account the effect of elevation and antenna patterns, which renders them unsuitable for the evaluation of future correlated 3D MIMO channels, currently being outlined in the next generation of standards. We derived the proposed SCF using SHE of plane waves and properties of Legendre and associated Legendre polynomials. This generalized function depends on the antenna patterns and angular densities through the FS coefficients of PAS and PES, which play an important role in characterizing the correlation. The interested users just need to provide these FS coefficients for any choice of angular distributions to obtain the correlation coefficients. Numerical results show an excellent agreement between the theoretical and simulated results. Therefore, our closed-form expression for the generalized SCF can be effectively used to evaluate the system performance of correlated 3D MIMO channels.

\section{REFERENCES}

[1] E. Telatar, "Capacity of multi-antenna Gaussian channels," European Trans. Telecommun., vol. 10, no. 6, pp. 585-595, Nov./Dec. 1999.

[2] G. J. Foschini, "Layered space-time architecture for wireless communication in a fading environment when using multi-element antennas," Bell Labs Technical Journal, vol. 1, no. 2, pp. 41-59, 1996.

[3] J. Koppenborg, H. Halbauer, S. Saur, and C. Hoek, "3D beamforming trials with an active antenna array," in Proc. ITG Workshop Smart Antennas, pp. 110-114, 2012.

[4] RP-122034, "Study on 3D channel model for elevation beamforming and FD-MIMO studies for LTE," 3GPP TSG RAN \# 58, Dec. 2012.

[5] A. Forenza, D. J. Love, and R. W. Heath, "Simplified spatial correlation models for clustered MIMO channels with different array configurations," IEEE Transactions on Vehicular Technology, vol. 56, no. 4, pp. 1924-1934, July 2007.

[6] W. J. L. Queiroz, F. Madeiro, W. T. A. Lopes, and M. S. Alencar, "Spatial correlation for DoA characterization using Von Mises, Cosine, and Gaussian distributions," International Journal of Antennas and Propagation, vol. 2011, July 2011.

[7] K. Mammasis and R. W. Stewart, "Spherical statistics and spatial correlation for multielement antenna systems," EURASIP Journal on Wireless Communications and Networking, vol. 2010, Dec. 2010.

[8] P. D. Teal, T. D. Abhayapala, and R. A. Kennedy, "Spatial correlation for general distributions of scatterers," IEEE Signal Processing Letters, vol. 9, no. 10, pp. 305-308, Oct. 2002.

[9] "Spatial Channel Model for Multiple Input Multiple Output (MIMO) Simulations," [Online]. Available: http://www.3gpp.org/ftp/Specs/ html-info/25996.htm, Sept. 2003.

[10] J. Meinilä, P. Kyösti et al., "D5.3: WINNER+ Final Channel Models V1.0," [Online.] Available: http://projects.celtic-initiative.org/winner+ /WINNER+\%20Deliverables/D5.3 v1.0.pdf, June 2010.

[11] Report ITU-R M.2135, "Guidelines for evaluation of radio interface technologies for IMT-advanced," [Online]. Available: http://www.itu.int/ pub/R-REP-M.2135-2008/en, 2008.

[12] A. Kammoun, H. Khanfir, Z. Altman, M. Debbah, and M. Kamoun, "Preliminary results on 3D channel modeling: From theory to standardization," IEEE Journal on Selected Areas in Communications, vol. 32, no. 6, pp. 1219-1229, June. 2014.

[13] K. Kalliola, K. Sulonen, H. Laitinen, O. Kivekas, J. Krogerus, and P. Vainikainen, "Angular power distribution and mean effective gain of mobile antenna in different propagation environments," IEEE Transactions on Vehicular Technology, vol. 51, no. 5, pp. 823-838, Sept. 2002.

[14] D. Colton and R. Kress, Inverse Acoustic and Electromagnetic Scattering Theory, 3rd ed. New York, NY: Springer, 2013.

[15] R. A. Kennedy and P. Sadeghi, Hilbert Space Methods in Signal Processing. Cambridge, UK: Cambridge University Press, 2013.

[16] D. J. Hofsommer and M. L. Potters, "Table Of Fourier Coefficients of Assosciated Legendre Functions," R 478 Computation Department of the Mathematical Center, Amsterdam, June 1960.

[17] T. Abhayapala, T. Pollock, and R. Kennedy, "Characterization of 3D spatial wireless channels," in Proc. IEEE Vehicular Technology Conference (VTC 2003-Fall), vol. 1, Oct. 2003, pp. 123-127.

[18] J. Zhang, C. Pan, F. Pei, G. Liu, and X. Cheng, "Three-dimensional fading channel models: A survey of elevation angle research," IEEE Communications Magazine, vol. 52, no. 6, pp. 218-226, June. 2014.

[19] G. Kurz, I. Gilitschenski, and U. D. Hanebeck, "Recursive Nonlinear Filtering for Angular Data Based on Circular Distributions," in Proceedings of the 2013 American Control Conference (ACC 2013), Washington D. C., USA, Jun. 2013. 